\title{
Accelerating distributed deep neural network training with pipelined MPI allreduce
}

\author{
Adrián Castelló $^{1}$ (D) Enrique S. Quintana-Ortí ${ }^{1} \cdot$ José Duato $^{1}$
}

Received: 25 January 2021 / Revised: 1 June 2021 / Accepted: 24 July 2021 / Published online: 7 August 2021

(C) The Author(s) 2021, corrected publication 2021

\begin{abstract}
TensorFlow (TF) is usually combined with the Horovod (HVD) workload distribution package to obtain a parallel tool to train deep neural network on clusters of computers. HVD in turn utilizes a blocking Allreduce primitive to share information among processes, combined with a communication thread to overlap communication with computation. In this work, we perform a thorough experimental analysis to expose (1) the importance of selecting the best algorithm in MPI libraries to realize the Allreduce operation; and (2) the performance acceleration that can be attained when replacing a blocking Allreduce with its non-blocking counterpart (while maintaining the blocking behaviour via the appropriate synchronization mechanism). Furthermore, (3) we explore the benefits of applying pipelining to the communication exchange, demonstrating that these improvements carry over to distributed training via TF+HVD. Finally, (4) we show that pipelining can also boost performance for applications that make heavy use of other collectives, such as Broadcast and Reduce-Scatter.
\end{abstract}

Keywords Message Passing Interface (MPI) - Collective communication primitives - Allreduce - Deep learning · Distributed training

\section{Introduction}

The outburst of deep learning (DL) technologies in the past few years has been accelerated by the development of efficient frameworks for distributed training of deep neural networks (DNNs) on clusters. Most of these frameworks exploit data parallelism (DP), by partitioning (and distributing) the workload among the cluster nodes/processes across the batch dimension (i.e., the inputs or samples) [5]. In this scenario, at each iteration of training, all processes collaborate to perform a reduction of the local weights in order to produce a global update of the parameters that define the DNN model [5, 8]. This synchronous version of training thus ensures that, prior to the next training iteration

\footnotetext{
Adrián Castelló

adcastel@disca.upv.es

Enrique S. Quintana-Ortí

quintana@disca.upv.es

José Duato

jduato@disca.upv.es

1 Universitat Politècnica de València, Valencia, Spain
}

(with a new batch of samples), all processes unite their state.

Data communication through the interconnection network is particularly crucial for the efficient synchronous DP training of convolutional neural networks (CNNs) on clusters of computers [8, 15]. Furthermore, data movements (across the memory hierachy as well as the nodes of a distributed platform) are a major source of energy consumption [26].

MPI (Message Passing Interface) [28] is the de facto standard for distributed high performance computing (HPC) applications. Therefore, it has been naturally adopted as the communication layer for distributed training frameworks such as Google's TensorFlow (TF) [1], TF+Horovod (HVD) [25], and PyTorch [24]. The MPI application programming interface (API) comprises a large variety of peer-to-peer and collective communication primitives. Among these, the DP scheme for distributed DNN training basically relies on the blocking MPI_Allreduce primitive, which internally reduces a collection of local values broadcasting the global result to all processes participating in the communication. 
In a previous work [6], we analyzed the impact on performance of selecting the best algorithmic realization of MPI_Allreduce, depending on the message size and cluster configuration, when leveraging TF+HVD to train CNNs on a small cluster equipped with an Infiniband network. In this paper we pursue the pipelined optimization of this global blocking reduction via the split of the data exchange into a collection of smaller MPI_Iallreduce calls, which offers a better overlap of computation with communication yielding a better utilization of the network bandwidth. In particular, our present work makes the following contributions:

- We perform a complete performance evaluation of a blocking global reduction when replacing the conventional (blocking) MPI_Allreduce call by the alternative non-blocking MPI_Iallreduce primitive immediately followed by the corresponding blocking synchronization MPI_Wait.

- We propose two realizations of MPI_Iallreduce that offer significantly higher performance when applied to perform a blocking global reduction. These implementations operate by dividing the message (transparently to the user) into either a collection of messages of a specific smaller size or a fixed number of smaller messages, in both cases pipelining the transfers.

- We demonstrate that the performance acceleration from pipelining the global reduction carry over to the distributed training of representative $\mathrm{CNNs}$ using the TF+HVD framework, offering performance improvements that vary between 5 and $60 \%$.

- We show that splitting the messages so as to pipeline the transfers benefits not only the global reduction primitive but also other collective operations, such as broadcast or reduce-scatter, which can improve performance for applications that make significant use of them.

- Finally, our complete experimental analyses employ three popular instances of MPI and explores the distinct algorithms for the MPI_Iallreduce primitive in those to detect the best operation depending on the number of nodes and message size. Furthermore, we conduct the experiments on a couple of recent Infiniband network technologies: QDR and EDR.

The rest of the paper is organized as follows. In Sect. 2 we provide a brief review of related work. In Sect. 3 we include a discussion of some popular algorithms for implementing a global reduction together with their theoretical costs. In Sect. 4 we conduct an analysis of these algorithms, and in Sect. 5 we introduce our pipelined optimizations for the MPI_Allreduce collective communication primitive. In Sect. 6 we extend the pipelining technique to other popular collective communication primitives. Section 7, we apply these optimizations to the TF+HVD DL environment. Finally, Sect. 8 summarizes the conclusions of this work.

\section{Related work}

\subsection{MPI collective communication primitives}

Since its initial appearance in the early nineties, MPI [10] has evolved to integrate new functionality in addition to many optimizations. One relevant example is the design of efficient algorithms for collective communication primitives (CCPs) in line with [9, 30]. In particular, the former work 1) formalizes the theoretical analysis of CCP, focusing on simple and effective solutions that generalize to multidimensional meshes and hypercubes; and 2) shows how the algorithms for a given CCP can be organized into parameterized families, which then expose the keys for performance. In the latter work, the authors propose new algorithms to improve the performance of CCPs, for clusters connected by switched networks, pursuing either the minimization of latency for short messages or the reduction of bandwidth use for long messages.

Other work aimed at improving the CCPs performance act at node level. In particular, in [21, 22], shared-memory is exploited to boost intra-node communication; and in [33] the reductions are performed by means of AVX-512 instructions.

There exist a few works that specifically evaluate and/or improve the MPI CCPs for DL, for example, taking into account the special characteristics of the messages that are exchanged in this type of applications [3, 4, 18, 23]. In addition, MPI-based software has been developed for distributed DNN training; for example, MVAPICH2-GDR ${ }^{1}$ from Ohio State University or oneAPI ${ }^{2}$ from Intel.

\subsection{Pipelining for MPI CCPs}

MPI was originally conceived with a strong focus on the efficient exchange of small messages. In consequence, the adoption of MPI in DL forced developers to study how to reduce the overhead of MPI for large messages. A step in this direction consists in dividing the transfers into a collection of smaller messages, yielding a pipelined (or segmented) communication scheme. In [32], the authors performed a manual segmentation for MPI_Reduce, MPI_Bcast, and MPI_Allreduce when communicating data among a few graphics processing units (GPUs) in the same node. In [31], the authors presented a pipelined

\footnotetext{
${ }_{1}$ https://mvapich.cse.ohio-state.edu/

2 https://github.com/oneapi-src
} 
data transfer mechanism for processes running on the CPUs of a single node. In two recent works [16, 17], the authors present a pipelining approach for two CCPs: MPI_Al1gather and MPI_Allgatherv with message sizes up to 64 and $8 \mathrm{MB}$, respectively, in a cluster.

In [2], a variant of pipelining is obtained by slicing the network (by means of virtual LANs) in order to exploit the full network bandwidth for data broadcasting.

Compared with previous work, we address the application of pipelining to optimize a blocking Allreduce, for very large messages, of up to $1 \mathrm{~GB}$, in the context of distributed DNN training on clusters of computer nodes. In addition, we demonstrate that the same technique renders appealing benefits for some collective primitives, such as MPI_Bcast and MPI_Reduce_scatter, but not for other cases, such as MPI_Allgather, due to their implementation in current MPI libraries.

\section{$3 \mathrm{MPI}$ algorithms for allreduce}

The term "reduction" is frequently used in DL and DNN frameworks to refer to the global update of the DNN model parameters (i.e., weights and biases) that is necessary at each iteration of the synchronous version of the training process. In practice, this reduction is performed using the conventional (blocking) MPI_Allreduce CCP which, depending on the MPI library, is realized via different algorithms [9, 30]. In this section we review some of the most common algorithmic realizations of MPI_Iallreduce, together with their theoretical cost. We note that the non-blocking primitive can be easily leveraged to mimic the behaviour of the blocking-counterpart, by simply adding a proper synchronization after it.

\subsection{A family of algorithms}

There exist a number of instances of the MPI library, with some prominent examples being OpenMPI, ${ }^{3} \mathrm{MPICH},{ }^{4}$ MVAPICH, ${ }^{5}$ and Intel MPI. ${ }^{6}$ All these implementations adhere to the functionality and specification defined by the MPI API, while distinct realizations of the standard vary in the implementation of the primitives and, quite often, the performance they attain.

The MPI instances usually optimize the CCPs via the implementation of a variety of algorithms (or communication schemes), in principle selecting the most appropriate

\footnotetext{
3 https://www.open-mpi.org

${ }^{4}$ https://www.mpich.org

5 https://mvapich.cse.ohio-state.edu

${ }^{6}$ https://software.intel.com/content/www/us/en/develop/tools/oneapi/ components/mpi-library.html
}

option at execution time depending, for example, on the message size, number of processes, network topology, etc. For the particular case of (the non-blocking) MPI_Iallreduce, the following list briefly describes some of the most popular algorithms (see [9, 12, 30] for additional details):

1. RDB (Recursive doubling): Initially, the processes that are a "distance" 1 apart (i.e., with rank identifiers that differ only by 1) exchange (and reduce) their data. Next, the processes that are a distance 2 apart do the same with the complete data they own after the first exchange. This is repeated for processes which are at distances $4,8, \ldots$ apart, till all processes have received (and reduced) all the data.

2. RSA (Rabenseifner's algorithm): This algorithm performs a Reduce-Scatter exchange followed by an Allgather. For this, the algorithm uses a combination of recursive-vector halving and recursive-distance doubling for the Reduce-Scatter stage, and recursivedoubling for the subsequent Allgather.

3. RNG (Ring): The message size is divided into one segment per process and each process then sends its segment to the next process, where it is reduced with the local data. Once this step is complete, the process is repeated $p-1$ times. Finally, an Allgather is applied.

4. BIN (Binomial tree): The processes first perform a common (reverse) binary (or binomial) tree-based reduction to a specific process, to then broadcast the result back to all processes using a binary tree-based broadcast.

The above-mentioned algorithms are not exclusive of MPI_Ial1reduce. In particular, its blocking counterpart is frequently implemented using the same algorithms (together with other options) [6]. Nonetheless, one major difference between the blocking and non-blocking variants is that, in MPI_Iallreduce, once all operations (communication and computation) are pushed to the scheduler, the control immediately returns to the user's application process. In comparison, for the MPI_Allreduce case, this control is only returned when all the operations are completed by the current process. A second difference lies in the specific peer-to-peer primitives that are utilized in each case.

\subsection{Theoretical cost analysis}

Let us consider a collection of $n \cdot p$ data items, evenly distributed across a platform consisting of $p$ nodes, with a single MPI process running on each node. Furthermore, consider the link latency is given by $\alpha$ (in seconds) and assume the link bandwidth, denoted by $\beta$ (in data items per second), is independent of the message size. Assume also 
that the interconnection network supports simultaneous transfers between all pairs of nodes at full link bandwidth. Finally, consider that each node can perform $\gamma$ arithmetic operations per second. Table 1 then displays the theoretical cost of the afore-described algorithms for MPI_Iallreduce separated into their latency, bandwidth, and arithmetic components. For simplicity, in the table we assume that $p$ is a power of 2. Otherwise, all logarithmic costs need to be rounded up to the nearest integer. When the message is large, as it is generally the case in the DNN training, the transfer cost is dominated by the bandwidth term and, therefore, the expressions in the table indicate that the best options are RSA and RNG.

\section{Performance analysis of MPI_Iallreduce}

MPI is nowadays widely adopted for distributed programming. However, our experience with several MPI libraries is that the algorithms which are automatically selected to execute the CCPs are often suboptimal for a considerable range of message sizes and number of nodes [6]. One main reason is that, while the theoretical cost models in Table 1 a priori identify the best algorithm(s), the experimental results may differ significantly for a number of causes. At this point, we recognize that most MPI instances were implemented with the target of optimizing the exchange of small messages on a reduced (usually, an integer power of two) number of nodes. This does not reflect the usual scenario in the case of DNN training.

In this section we conduct a complete evaluation of the algorithms for MPI_Iallreduce in three well-know, widely-used MPI libraries, and compare the available options with the automatic selection made by the library (hereafter referred to as AUTO), so as to asses if it selection is properly done.

\begin{tabular}{|c|c|c|c|c|}
\hline Id. & Alg. & Latency $\times \alpha$ & Bandwidth $\times \beta^{-1}$ & Arithmetic $\times \gamma^{-1}$ \\
\hline 1 & $\mathrm{RDB}$ & $\log p$ & $n \log p$ & $n \log p$ \\
\hline 2 & RSA & $2 \log p$ & $2 n \frac{p-1}{p}$ & $n \frac{p-1}{p}$ \\
\hline 3 & RNG & $2(p-1)$ & $2 n \frac{p-1}{p}$ & $n \frac{p-1}{p}$ \\
\hline 4 & $\mathrm{BIN}$ & $2 \log p$ & $2 n \log p$ & $n \log p$ \\
\hline
\end{tabular}

\subsection{Experimental setup}

For our evaluation, we employ OpenMPI 4.1.0rc, MPICH 3.3.1, and Intel MPI 2020. The cluster platform for these experiments consists of 9 nodes equipped with 2 Intel Xeon Gold 5120 CPUs (14 cores each, for a total of 28 cores per node) at $2.20 \mathrm{GHz}, 192 \mathrm{~GB}$ of DDR4 RAM, and an NVIDIA V100-PCIe GPU with 32 GB of HBM2 memory. The nodes are connected via an InfiniBand EDR network with a link latency of $0.5 \mu$ s and a link bandwidth of $100 \mathrm{Gbps}$.

In all experiments in this paper, the performance reported for a given algorithm is calculated as the throughput rate that is obtained by dividing the size of the message $n$ (in bytes) with the time required to complete the global reduction. The tests are repeated a large number of times and the execution time corresponds to the average cost. As we are dealing with non-blocking primitives, each call is paired with its corresponding synchronization (MPI_Wait) in order to ensure that the execution is complete prior to measuring the execution time. About the selected metric, on the negative side, the actual number of transferred bytes during the reduction is considerably larger. Therefore, the transfer rate represented with this metric is in general quite below the actual network bandwidth. On the positive side, the metric is inversely proportional to the execution time for all algorithms and libraries and, therefore, sets the grounds for a fair comparison of these.

\subsection{Analysis}

In Fig. 1, we display the throughput rates (measured in millions of bytes per second, or $\mathrm{MB} / \mathrm{s}$ ) attained by the algorithms for MPI_Iallreduce. This comparison includes the distinct algorithms in OpenMPI, MPICH, and Intel MPI, and is conducted using 8 and 9 MPI processes/ nodes. (The latter configuration is chosen to evaluate the effect of executing the algorithms with a number of nodes that is not an integer power of two.)

The first factor exposed by the charts in Fig. 1 is the distinct number of algorithms depending on the specific MPI library: While OpenMPI and MPICH integrate a reduced set of options (4 and 5, respectively), Intel MPI spans a much larger number of possibilities (a total of 9).

This study reveals that the best option is in general given by the RNG algorithm in OpenMPI. Although Intel MPI also implements that particular algorithm, it does not achieve a comparable throughput. In contrast, MPICH does not offer a realization of this algorithm. Analyzing the AUTO selections, all three MPI instances select RSA (one of the two best option for large messages, from the theoretical point of view; see Table 1). However, this choice 

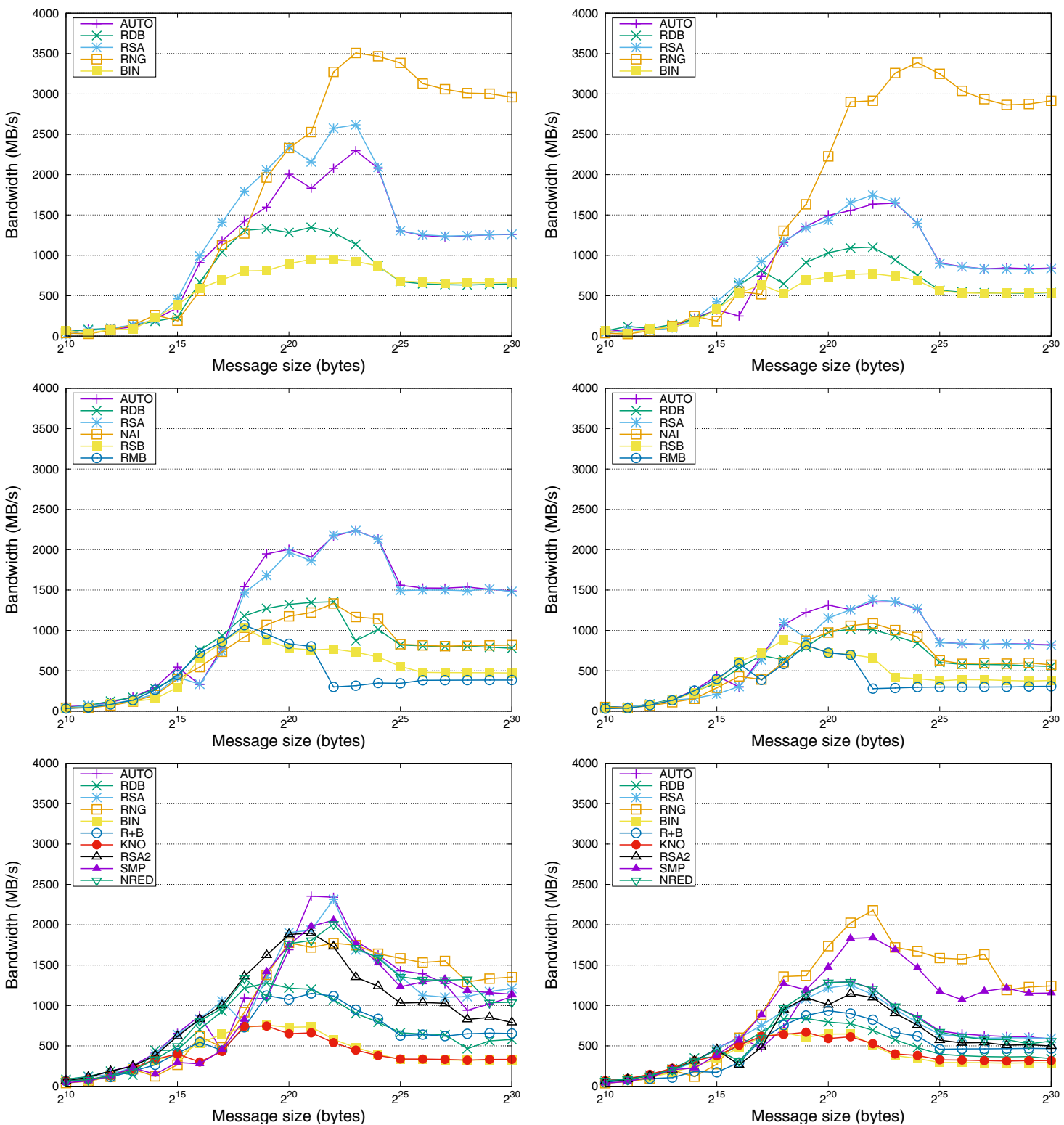

Fig. 1 Performance of the algorithms in OpenMPI, MPICH and Intel MPI for MPI_Iallreduce (top, middle and bottom, respectively) using 8 and 9 nodes/processes (left and right, respectively)

corresponds to the actual optimal option only for MPICH. This experiment clearly shows that a correct selection of the algorithm can significantly improve performance. Taking into account the considerably higher performance attained with OpenMPI, for brevity in the remainder of the paper we exclude MPICH and Intel MPI from the discussion. The complete results using these two other libraries can be found in Appendix A.

\section{Pipelined MPI_Iallreduce}

Although the MPI_Iallreduce CCP is usually viewed as a "monolithic" operation, its realization comprises a sequence of intertwined communication exchanges and arithmetic computations. Specifically, this CCP consists of a set of calls to the non-blocking peer-to-peer primitives in the MPI API for sending and receiving data, MPI_Isend and MPI_Irecv respectively, plus a few simple arithmetic computations. All these are passed to a communication runtime scheduler (and the associated thread) so that the application thread which invoked MPI_Iallreduce 
can continue with the execution of the user's code while the non-blocking data transfers are performed.

The possibility of overlapping communication and computation, in addition to a potential infra-utilization of the network bandwidth, offer appealing niches for performance improvement, especially for large messages. In this section, we explain how addressing these weakness via the concurrent execution of multiple MPI_Iallreduces improves performance.

\subsection{Pipelining with fixed message size}

In order to obtain a pipelined variant of the global reduction, we can divide (that is, partition or segment) the original exchange into several non-blocking calls, as shown in Listing 1. There, we split the message, consisting of $\mathrm{s}$ bytes, into several segments (or chunks) of segsize bytes each (except, possibly, for the last one), and perform numseg $=\lceil\mathrm{s} /$ segsize $\rceil$ consecutive invocations to the non-blocking MPI_Iallreduce CCP, that is one per segment, to initiate the exchange of the corresponding segment. Note that the routine returns the number of segments so that a conventional blocking behaviour can be achieved by simply invoking the synchronizing MPI_Waitall.

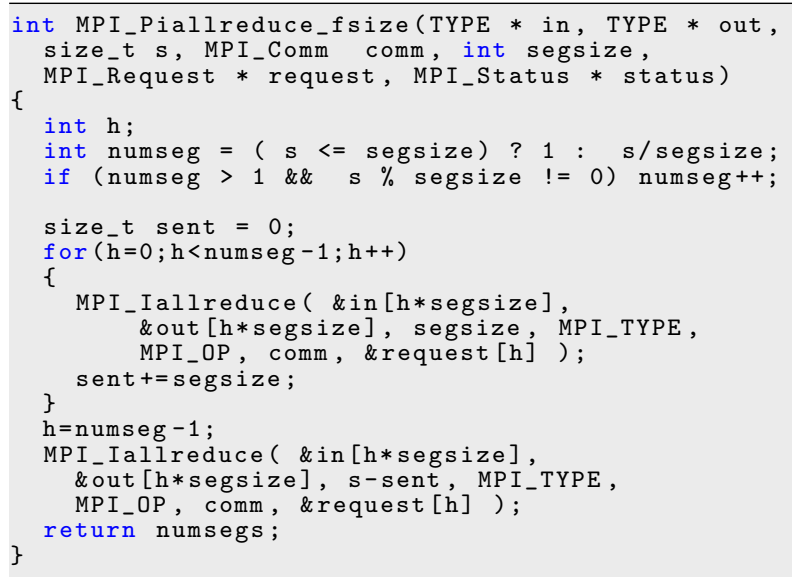

Figure 2 reports the effect of pipelining with a fixed message size, applied to the AUTO and RNG algorithms for both 8 and 9 nodes. For the AUTO option (RSA for OpenMPI), these results show a performance gain for the pipelined variants of about $250 \mathrm{MB} / \mathrm{s}$ for message sizes larger than $32 \mathrm{MB}$ and 8 processes. From that size and beyond, dividing the message into $32 \mathrm{MB}$ segments is one of the best options. For AUTO and 9 processes, the performance gain of the pipelined realizations is slightly higher: from $800 \mathrm{MB} / \mathrm{s}$ up to more than 1,200 MB/s. For the best algorithm (RNG for OpenMPI), the pipelined variants generally outperform AUTO by a factor of $2 \times$. In addition, dividing the message into several segments allows to maintain the asymptotic exchange throughput at $4,000 \mathrm{MB} / \mathrm{s}$, whereas the original primitive falls from a peak of $3,500 \mathrm{MB} / \mathrm{s}$ to $3,000 \mathrm{MB} / \mathrm{s}$ or less for large messages.

\subsection{Pipelining with a fixed degree of concurrency}

An alternative to obtain a pipelined realization of MPI_Iallreduce is to divide the message into a fixed number of smaller messages. For that purpose, we employ the code in Listing 2, where we perform the global reduction of a message, of $s$ bytes, by means of numseg calls to the non-blocking MPI_Iallreduce CCP, one per segment of segsize $=\mathrm{s} /$ numseg bytes (except for the last one, which has to take into account the possibility of the message size not being an integer multiple of the number of segments). Note that the number of primitives which are concurrently executed in this scheme is fixed. The routine also returns the number of segments so that a blocking behaviour to allow a straight-forward realization of a blocking behaviour via the invocation to MPI_Waitall.

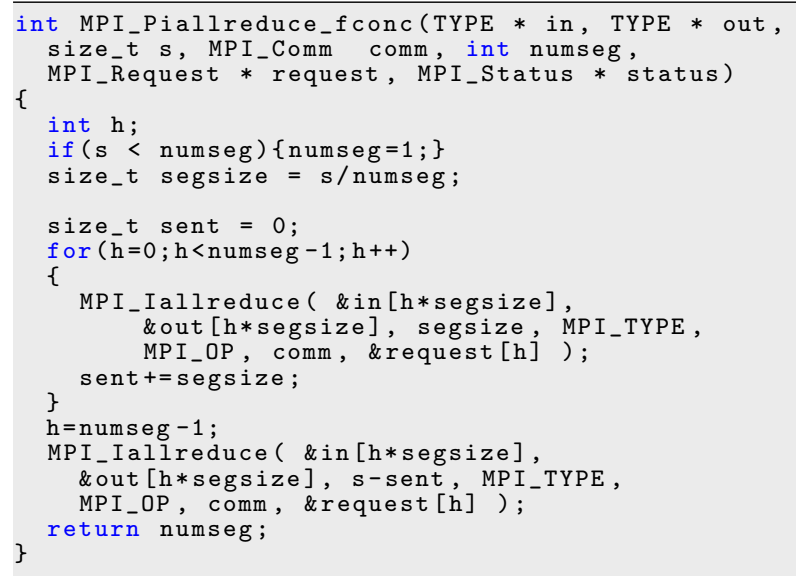

Figure 3 illustrates the impact of pipelining with a fixed degree of concurrency. The two top charts in the figure confirm that the AUTO algorithm (for OpenMPI, RSA) only benefits from this type of pipelining for messages of size larger than $32 \mathrm{MB}$. In contrast, the pipelined RNG algorithm (see the bottom two charts) already improves the performance for messages of size larger than $2 \mathrm{MB}$. In addition, these charts expose that it is possible to accelerate the throughput by up to $25 \%$ applying this type of pipelining.

Taking into account that the RNG algorithm outperforms AUTO by a factor of two, pipelining the RNG algorithm for large messages improves the communication 

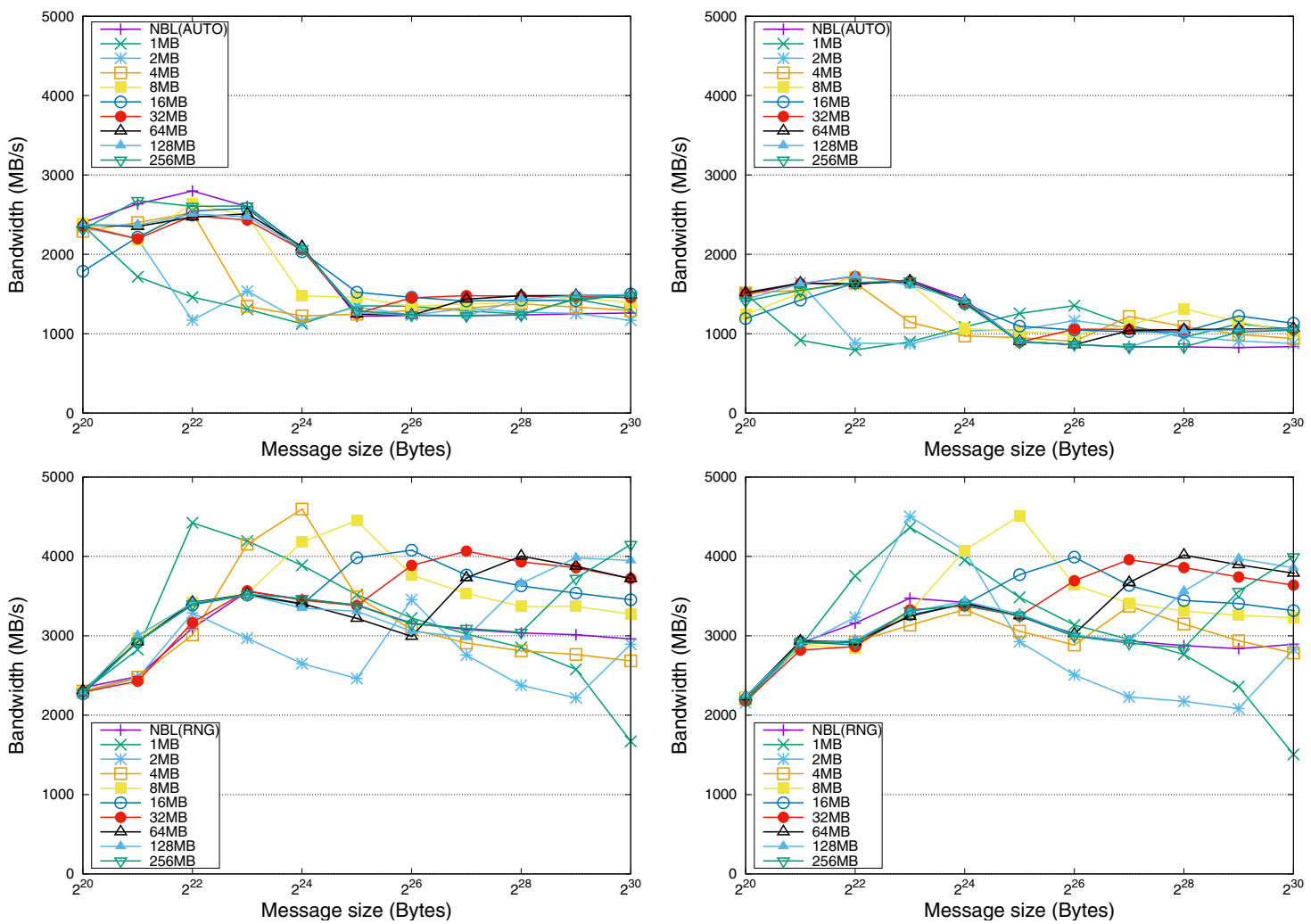

Fig. 2 Performance of the AUTO and RNG algorithms in OpenMPI (top and bottom, respectively) using 8 and 9 nodes/processes (left and right, respectively). The NBL(AUTO) and NBL(RNG) labels

performance of the automatic selection from 1,400 to $4,000 \mathrm{MB} / \mathrm{s}$ for 8 processes and from 800 to $4,000 \mathrm{MB} / \mathrm{s}$ for 9 processes.

Both pipelining techniques have demonstrated significant performance advantage with respect the conventional MPI_Iallreduce. However, when dealing with an application where the message may vary in size from one transfer to another (as it is the case of DNN training), it is more convenient to apply the pipelining with a fixed degree of concurrency. In this scenario, a solution based on a fixed-size segment may result in an elevate number of messages if the size is too small, constraining the performance as it is shown in Fig. 2. In consequence, in the remainder of this paper we will consider only the variant that applies pipelining with a fixed degree of concurrency.

\subsection{Identification of the source of gains}

We next present a simple experiment that demonstrates the communication-computation overlap that takes place when splitting the reduction primitive into multiple smaller calls. For this purpose, we have modified OpenMPI to eliminate the arithmetic computations that occur inside the MPI_Iallreduce primitive by omitting the submission

correspond to the evaluation of the original MPI_Iallreduce primitive; the labels of type XMB indicate the pipelined variant MPI_Piallreduce_fsize with segment size segsize=X MB

of the corresponding arithmetic tasks to the communication scheduler.

Figure 4 shows the throughput rate for the RNG algorithms with and without the arithmetic operations which are necessary for the reduction. The lines labeled with the NOOP suffix correspond to the modified OpenMPI routine without arithmetic operations. The lines with labels NBL and NBLx4 distinguish between the conventional and pipelined variants. The direct comparison between the lines with the NOOP suffix and the conventional variant (label "NBL(RNG)") exposes the contribution of the arithmetic to the cost of the reduction operation, which represents between 28 to $37 \%$ of the total execution time.

Now, by applying pipelining, the difference with respect to the NOOP versions is reduced, and the fraction of time with non-overlapped communication-computation diminishes to less than 14\%: compare the lines with the NOOP suffix and the pipelined variant (label "NBLx4(RNG)". In summary, although the pipelining does not totally overlap computation with communication, it reduces the impact of arithmetic on the global cost of MPI_Iallreduce significantly. 

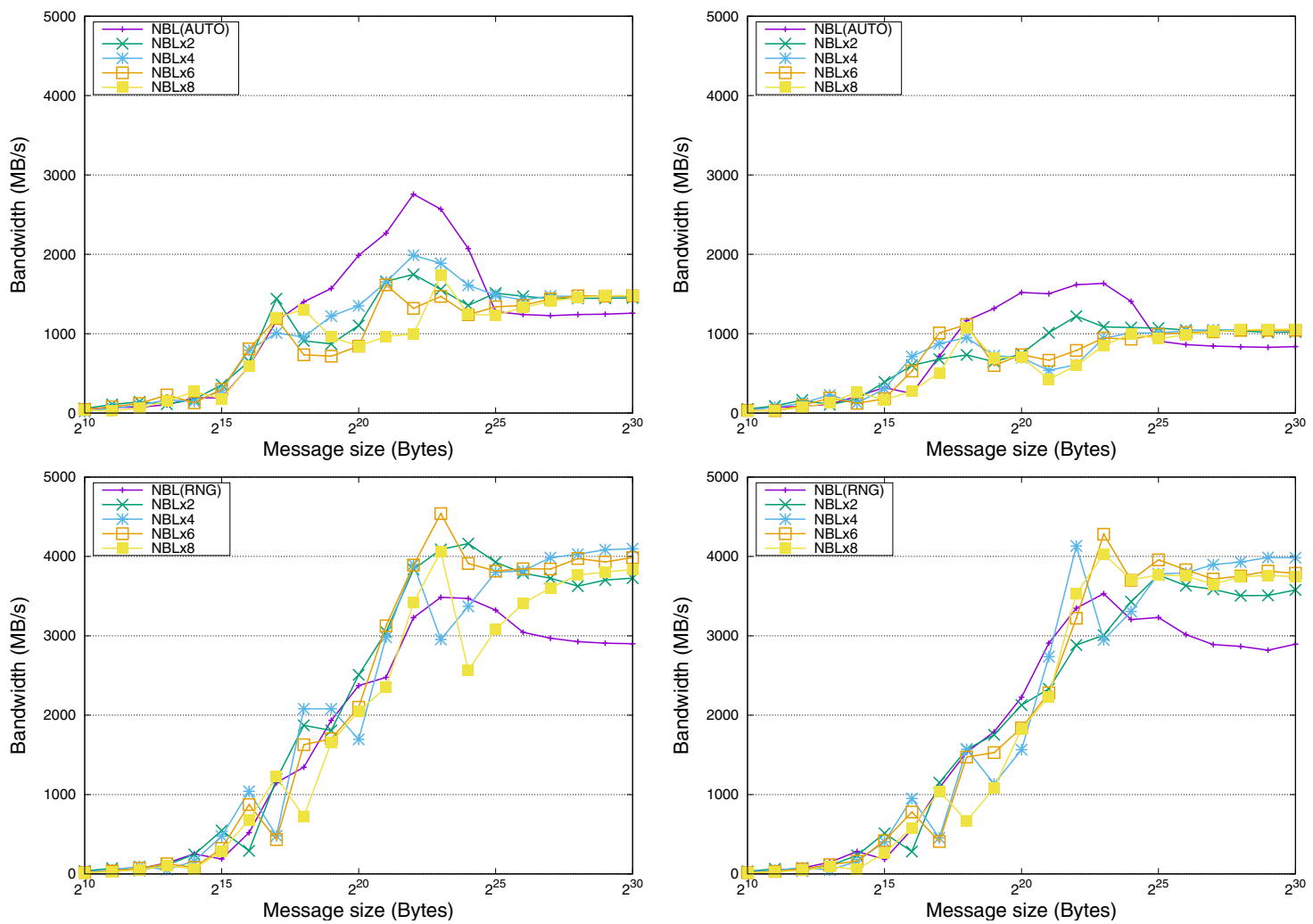

Fig. 3 Performance of the AUTO and RNG algorithms in OpenMPI (top and bottom, respectively) using 8 and 9 nodes/processes (left and right, respectively). The $\mathrm{NBL}(\mathrm{AUTO})$ and $\mathrm{NBL}(\mathrm{RNG})$ labels

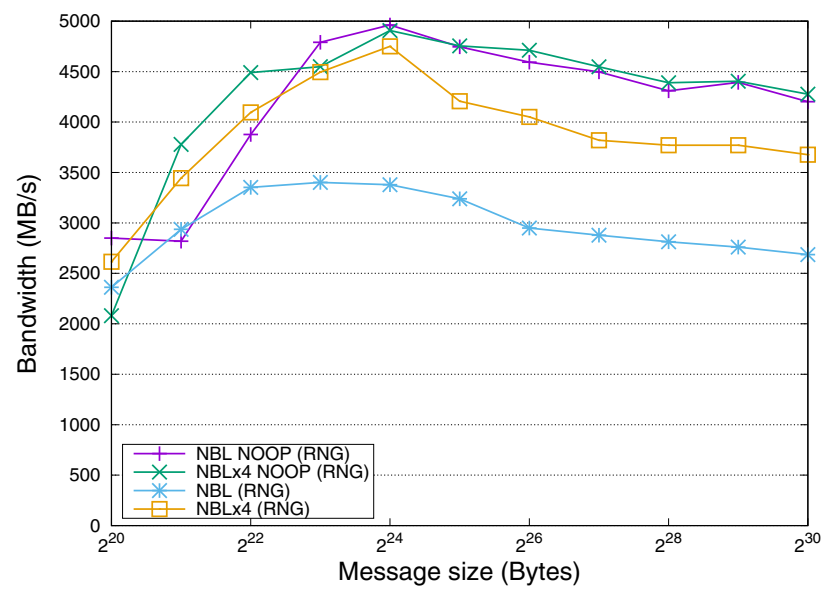

Fig. 4 Performance of the RNG algorithm in OpenMPI using 8 nodes/processes. The NBL NOOP suffix identifies the modified variant of MPI_Iallreduce without arithmetic operations; the labels of type NBL and NBLx4 respectively indicate the conventional variant MPI_Piallreduce_fconc and the pipelined alternative with numseg $=4$ segments

correspond to the evaluation of the original MPI_Iallreduce primitive; the labels of type NBLXY indicate the pipelined variant MPI_Piallreduce_fconc with numseg $=Y$ segments

\section{Extension of pipelining to other MPI collectives}

While our work was, so far, mainly focused in MPI_Allreduce, the MPI standard defines several other collective primitives that involve multiple processes inside a communicator. In this section, we evaluate the possibility of improving the performance of these other primitives by pipelining their data transfers.

\subsection{MPI_Allgather}

MPI_Allgather performs an all-to-all communication where each MPI process broadcasts its portion of the final result to the other processes. The logical communication pattern is thus similar to that present in MPI_Allreduce, as each MPI process contributes a piece of the final result, except in that there is no reduction performed during the data recollection.

Figure 5 shows the result of applying pipelining to the MPI_Iallgather collective. All variants there rely on a LIN-based algorithm, which turns out to be the best option for this primitive. Unfortunately, for this particular primitive applying, a pipelined communication scheme does not 

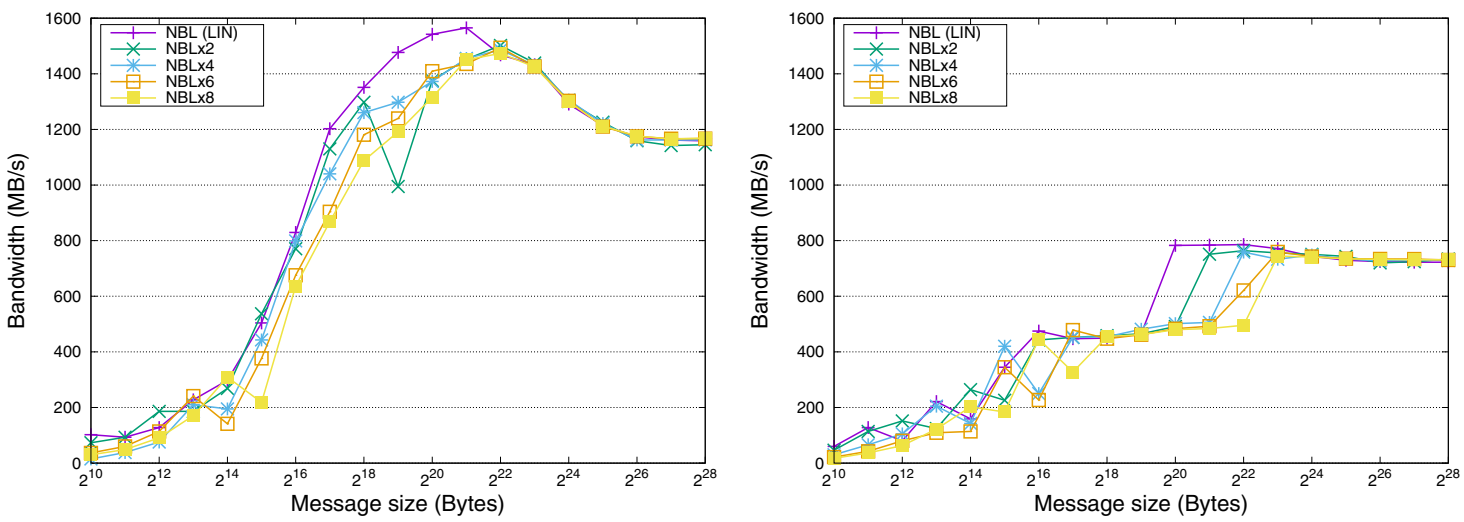

Fig. 5 Performance of the LIN algorithm for MPI_Iallgather in OpenMPI using 7 and 8 nodes/processes (left and right, respectively). The labels of type NBLxY indicate the pipelined variant MPI_Piallgather_fconc with numseg=Y segments

render any performance improvement. Even worse, in the case of $8 \mathrm{MPI}$ processes, the message pipelining reduces the performance for a certain range of message sizes. The reason for this lies in the particular "linear" implementation of this primitive in OpenMPI, which implies that segmenting the data transfers increments the number of messages but does not render a higher degree of parallelism among data transfers.

\subsection{MPI_Bcast}

MPI_Bcast is a popular collective primitive where a message is broadcast from one single process to all other processes participating in the communicator.

Figure 6 highlights the effect on performance of exploiting pipelining in the asychronous variant of this collective. In this case, the AUTO algorithm follows a CHAIN implementation which is already segmented internally. Nonetheless, adding an extra level of segmentation increases the performance from the initial 2,400 and $1,900 \mathrm{MB} / \mathrm{s}$ to 3,500 and $3,250 \mathrm{MB} / \mathrm{s}$ for 7 and 8 processes, respectively. In contrast with the
MPI_Iallgather case, for the broadcast the communication has a single original (root) process. In consequence, splitting the message into smaller chunks augments the number of concurrent data transfers.

\subsection{MPI Reduce scatter}

MPI_Reduce_scatter, as its name indicates, combines a first stage that reduces the contents of a data array into a single process, to then split (and scatter) the result of the reduction among all processes in the second stage.

Figure 7 displays the effect of applying a pipelined scheme to the asynchronous version of this collective. The results expose two different scenarios: For messages of size smaller than $16 \mathrm{MB}$ ( $2^{24}$ bytes), the best option uses a single segment (i.e., no pipelining). In contrast, for messages larger than that threshold, segmenting the communication improves the total performance by about $100 \mathrm{MB} /$ s. As was the case for MPI_Allreduce, the performance gain comes from the reduction stage, but the effect is mostly blurred by the scatter stage if the data size is small. Note that OpenMPI implements only one algorithm for this
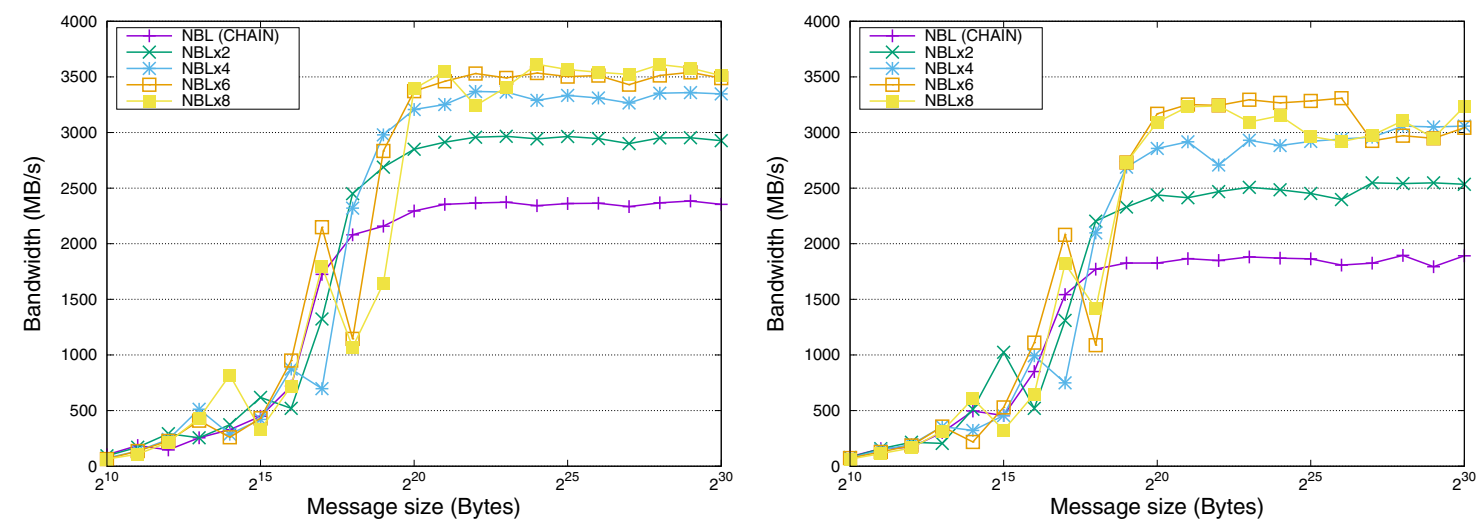

Fig. 6 Performance of the CHAIN algorithm for MPI_Ibcast in OpenMPI using 7 and 8 nodes/processes (left and right, respectively). The labels of type NBLxY indicate the pipelined variant MPI_Pibcast_fconc with numseg=Y segments 

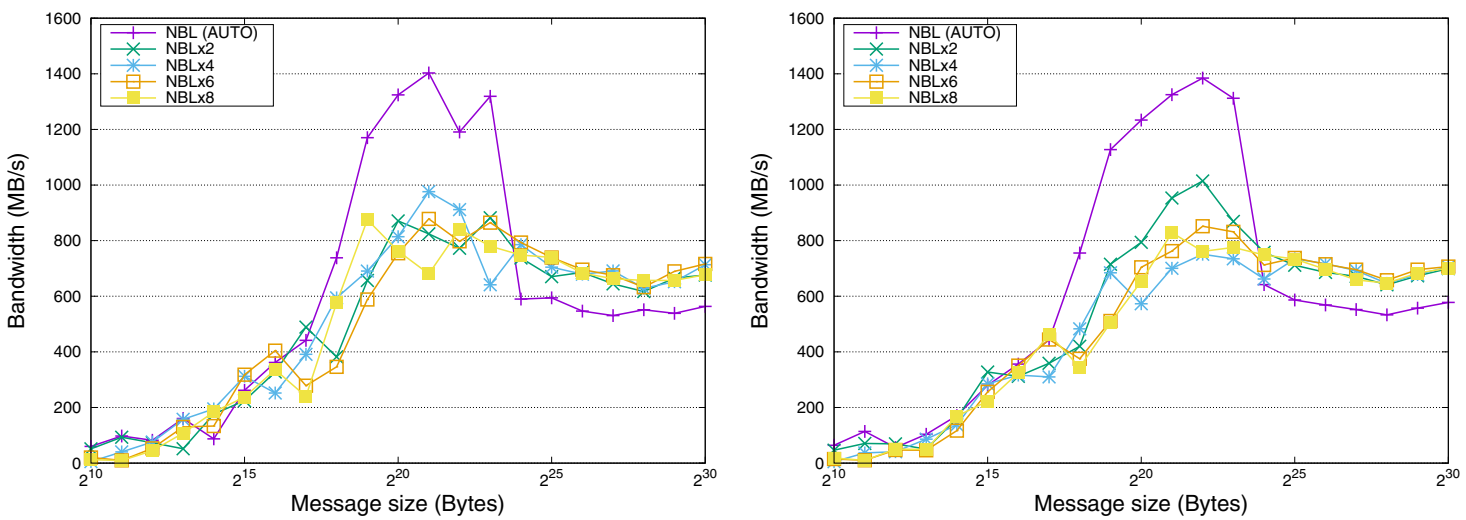

Fig. 7 Performance of the AUTO algorithm for MPI_Ireduce_scatter in OpenMPI using 7 and 8 nodes/processes (left and right, respectively). The labels of type NBLXY indicate the pipelined variant MPI_Pireduce_scatter_fconc with numseg=Y segments

primivite and, therefore, the AUTO option simply relies on that.

\subsection{Other collectives}

The MPI standard also defines other relevant collective primitives:

MPI_Alltoall,

MPI_Gather,

MPI_Scatter, and MPI_Reduce. In this work though, we do not consider them because of their similarities with the four analyzed primitives. Specifically, MPI_Al1toall and MPI_Gather are highly related with MPI_Allgather in the communication pattern; MPI_Scatter is a "reversed" realization of MPI_Gather, and therefore it is also linked with MPI_Al1gather; finally, MPI_Reduce is a "chopped" version of MPI_Allreduce, where there is a single reduction point and, given that the performance gains for the latter mostly come reduction part, we may expect a similar behaviour.

Some of the collective communication primitives reviewed in this section are heavily leveraged in distributed training of DNNs. Specifically, MPI_Allgather, MPI_Bcast, MPI_Reduce_scatter and MPI_Allreduce are employed for distributed DL frameworks that exploit model parallelism instead of the conventional data-parallel approach [7].

\section{Acceleration of distributed DNN training}

In this section we assess the benefits of the pipelined communication schemes proposed in our work when applied to accelerate distributed training of DNNs on a cluster of computer nodes, possibly enhanced with GPUs.

\subsection{Experimental training setup}

In order to illustrate the advantage of the new CCPs, we employ TF 2.1.0 with Horovod (HVD) 0.20.3 as the target framework for distributed CNN training. In the experiments, HVD is compiled and linked with OpenMPI 4.1.0 and CUDA support. For the evaluation involving GPUs, we also consider NVIDIA NCCL 2.7.8 as an alternative communication layer. For the computations, we use Intel MKL 2020 in the case of CPUs and CUDA 10.2 and cuDNN 8.0 for GPUs. All the results are obtained using the TF benchmark suite [14] executed with Python3.7.

To close this short review of the training setup, we consider a testbed consisting of four CNN models: AlexNet [20], ResNet50 [13], VGG11 [27], and ResNet110; and two datasets: Cifar10 [19] and ImageNet [11].

Table 2 characterizes each DNN model-dataset combination, indicating the total number of layers, the amount of model parameters, and the floating point operations (flops) per training iteration. (The latter parameters is actually a function of the batch size $b$.) For simplicity, we only report the number of flops for the forward pass; the total amount flops for a complete forward-backward iteration of training is roughly obtained by multiplying this number by 3 .

Table 2 Characterization of the DNN models+datasets. AlexNet, ResNet50 and VGG11 employ ImageNet and ResNet110 is for Cifar 10

\begin{tabular}{llll}
\hline Model & \# of layers & Params. & flops $\times b$ \\
\hline AlexNet & 22 & $62,378,344$ & $2,270,512,192$ \\
ResNet50 & 176 & $25,636,712$ & $8,178,368,512$ \\
VGG11 & 30 & $132,863,336$ & $15,218,180,096$ \\
ResNet110 & 393 & $1,747,898$ & $506,832,128$ \\
\hline
\end{tabular}




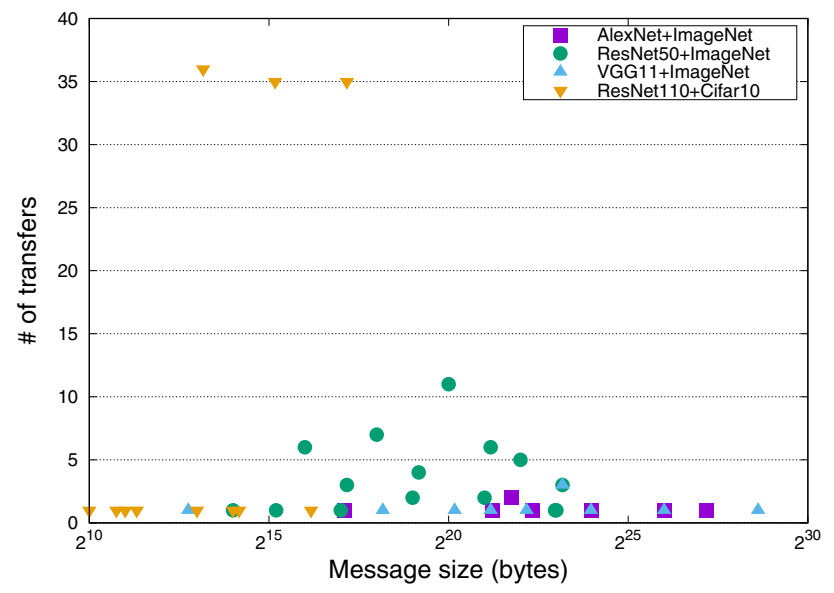

Fig. 8 Number of transfers clustered by message size for the DNN models+datasets

Figure 8 reports the number of messages and their size for the model-dataset pairs. We can clearly observe there that each scenario exhibits quite different transfer requirements. At this point it is necessary to point out that, in practice, frameworks such as HVD group several consecutive "small" data transfers into a single one to reduce latency. Therefore, the number of messages per forwardbackward pass may be smaller than the amount of convolutional and fully-connected layers for a DNN model. (Concretely, HVD groups small data transfers into $64 \mathrm{MB}$ data transfers.) Grouping messages into larger data transfers favors pipelining, as it increases performance for messages of that size.

\subsection{Brief discussion of TF+HVD}

At each iteration, HVD exploits DP by distributing a global batch size of $b \cdot p$ samples among the $p$ processes/nodes; TF then performs the local computations corresponding to the forward pass and backward propagation [29]; and HVD orchestrates the global reduction necessary for the update of the DNN model (that is, weight and biases) prior to the next iteration.

The original version of HVD relies on the blocking MPI_Allreduce primitive to perform the global reduction. Furthermore, HVD off-loads the execution of these primitives to a communication thread, which allows to overlap the operations that are necessary for the computational parts of the backward propagation (performed by the application processes/threads and TF) with the communication for the global reduction $[8,25]$.

In order to test our (non-blocking) pipelined $\mathrm{CCP}$, we developed a modified version of HVD that replaces the blocking collective with an adaptation of the code shown in Listing 2, followed by a wait synchronization. The numerical behavior and, therefore, the convergence rate of our $\mathrm{TF}+$ modified HVD version is equivalent to that of the original TF+HVD framework. In consequence, we can directly compare the execution time of the two solutions for a specific number of training epochs (10 in the experiments). To avoid variations, the framework is initialized with the same random seeds, yielding the same starting DNN model for the training process and, consequently, the same training convergence (except for the effect of rounding errors).

\subsection{Cluster of multicore processors}

Figure 9 illustrates the acceleration (or speed-up) attained when replacing the conventional invocation to (the blocking) MPI_Allreduce in HVD, with the blocking optimal algorithm for OpenMPI (that is, RNG), the nonblocking counterpart, and the pipelined variant (with the fixed concurrency degree set to 4). The local batch size $b$ for this particular experiment is set to either 32 or 64 . We note that, increasing the batch size generally reduces the contribution of the communication to the training cost per iteration, but may increase the total time due to a decay in the convergence rate.

The results in Fig. 9 offer three main conclusions: (1) The non-blocking MPI_Iallreduce outperforms its blocking counterpart, likely due to implementation decisions of the internal peer-to-peer calls; (2) the RNG algorithm offers considerably higher performance than the AUTO selection for this scenario; and (3) applying pipelining significantly improves the training performance of the framework.

In general, the performance gains vary depending on the contribution of communication to the total training cost, which in turn strongly depends on the CNN model (and, of course, the cluster hardware configuration). For example, applying the pipelining techniques to a communicationbound testbed, such as ResNet110 with Cifar10, improves the performance by a factor that is between 50 and $60 \%$. Conversely, when the computation dominates the total execution time (as, for instance, is the case for ResNet50 and VGG11 when trained with ImageNet), the performance gain is more modest, in the range between 5 and $22 \%$.

\subsection{Cluster of multicore processors with GPU accelerators}

In the last experiment, we investigate the impact of pipelining on the training throughput when the target cluster is equipped with graphics accelerators. For this particular case, in the comparison we also consider an alternative where the communication layer provided by NVIDIA's proprietary NCCL library instead of MPI. NCCL provides highly efficient primitives for Infiniband, 

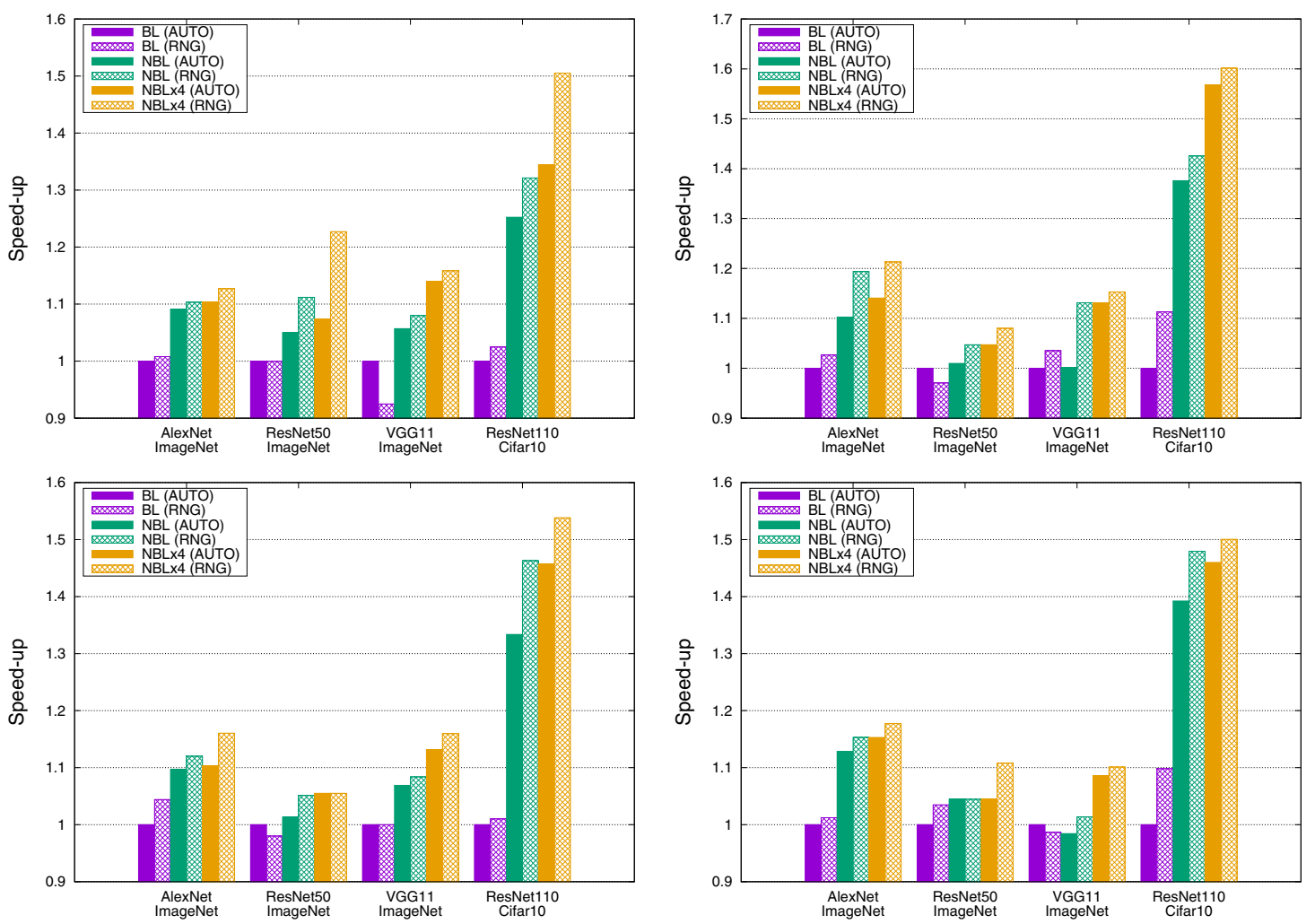

Fig. 9 Speed-up of TF+HVD when the blocking AUTO algorithm for MPI_Allreduce (BL(AUTO)) is replaced with either the blocking RNG algorithm (BL(RNG)), the non-blocking algorithms (NBL(AUTO/RNG)), or the pipelined variants of the latter

with direct access to the GPU memory and, therefore, is very difficult to outperform by a "general-purpose" library such as MPI. Nonetheless, we intend to verify whether it is possible to reduce the difference between MPI and NCCL by pipelining the communications.

Figure 10 reports the training throughput rate (meassured in terms of number of images processed per second, or images/s) and speed-up with respect the conventional blocking implementation in HVD based on MPI_Allreduce. In this study, we only consider the RNG algorithm since this has been identified as the best MPI-based option for this scenario. As it already occurred when the experiment did not exploit the GPUs, for those cases where the communication is the bottleneck (e.g., AlexNet and VGG11 with ImageNet), NCCL clearly outperforms any of the MPI configurations. Conversely, for compute-bound training scenarios (e.g., ResNet50 with ImageNet and ResNet110 with Cifar10 and a large batch size), the difference between NCCL and MPI is negligible. The performance improvement when pipelining is applied yields a speed-up of up to $60 \%$ in the best case. If we compare the pipelined MPI_Iallreduce version against NCCL, we observe that it performs close to the NVIDIA solution, with the difference narrowing as the batch size is

(NBLx4(AUTO/RNG)), using batch sizes of 32 and 64 (top and bottom, respectively), and 8 and 9 nodes/processes (left and right, respectively)

increased. At this point we repeat that augmenting the batch size may affect the convergence and accuracy of the training process, which often asks for a very fine-grain, application-dependent, tuning of the learning rate that needs to be dynamically varied as the training process evolves. Therefore, this is a complex technique which requires special knowledge and care.

\section{Conclusions}

We have reported notable improvements on the performance of the blocking Allreduce via 1) an adequate selection of the underneath algorithm;2) the use of its nonblocking counterpart followed by a synchronization primitive, to preserve the global blocking behaviour; and 3) pipelining the original call into a collection of smaller collectives that accommodates a better overlap of computation with communication, yielding a better utilization of the network bandwidth. In general, the RNG algorithm tends to be the best Allreduce option for the target dataparallel TF+HVD framework but this is rarely selected as default algorithm and, in the case of MPICH, not even implemented. Furthermore, we have demonstrated that the 

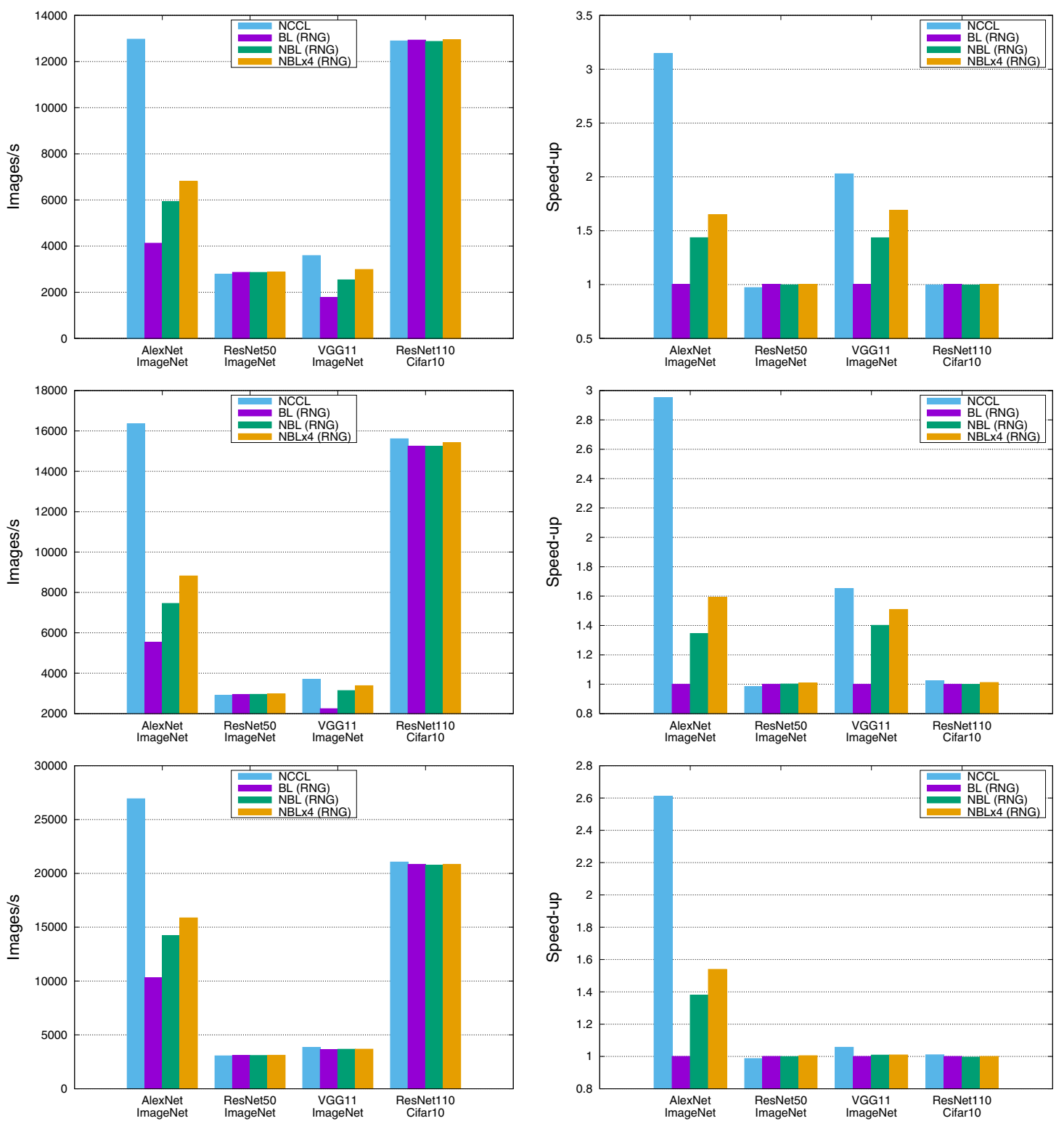

Fig. 10 Training throughput (in images/s) and speed-up (left and right, respectively) of TF+HVD with 8 GPUs/nodes when the blocking AUTO algorithm for MPI_Allreduce (BL(AUTO)) is replaced with either the blocking RNG algorithm (BL(RNG)), the non-blocking algorithms (NBL(AUTO/RNG)), or the pipelined

performance advantages of the segmentation/pipelining techniques carry over to other relevant collective primitives.

These benefits have been demonstrated for MPI via a experimental analysis but, more importantly, also for a relevant framework for distributed training of DNNs: $\mathrm{TF}+\mathrm{HVD}$. For those training testbeds where the communication plays a key role, on clusters of multicore processors, the proposed optimizations of the MPI layer yield an acceleration of the training performance for $\mathrm{TF}+\mathrm{HDV}$ of up to $22 \%$ for AlexNet and ResNet50; $15 \%$ for VGG11;

variants of the latter (NBLx4(AUTO/RNG)), using batch sizes of 96, 128 and 256 (top, middle and bottom, respectively). For reference, we also include the results when the communication layer is provided by NCCL

and $50-60 \%$ for ResNet110 with respect to the configuration automatically selected by MPI. For platforms equipped with GPUs, NVIDIA's NCCL is still offers the best communication layer, outperforming any of the MPI-based solutions. However, the techniques that have been proposed in this work help to close the performance gap between NCCL and MPI by a significant margin. In general, we can expect that the segmentation/pipelining approach will benefit many distributed applications that makes heavy use of collective primitives. 

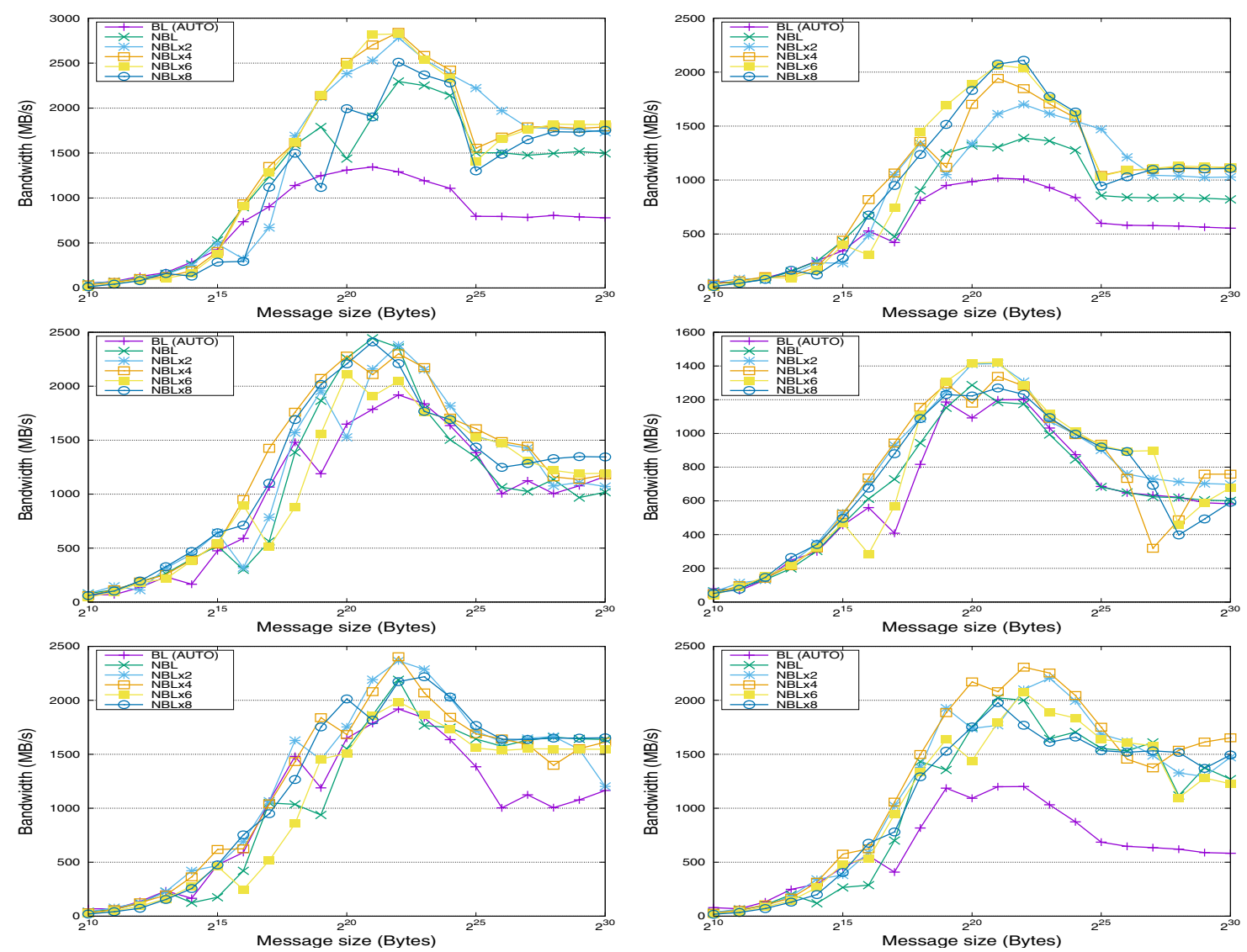

Fig. 11 Performance of the AUTO algorithm in MPICH (top), and the AUTO and RNG algorithms in Intel MPI (middle and bottom, respectively) using 8 and 9 nodes/processes (left and right, respectively)

\section{Complementary experiments}

In this appendix, we assess the benefits of pipelining for a variety of experimental configurations: MPI library, network technology, network topology, and processor family. In all plots, the BL(AUTO), NBL(AUTO) and NBL(RNG) labels correspond to the evaluation of the conventional MPI_Allreduce collective and the original (nonblocking) MPI_Iallreduce; the labels of type NBLxY indicate the pipelined variant MPI_Siallreduce_fconc with numseg $=Y$ segments.

\section{MPI libraries}

The target platform and libraries utilized in this first subsection are the same described in Sect. 7.1.

Figure 11 shows that the performance gains attained with pipelining are considerable for both the AUTO and BEST algorithms in MPICH and Intel MPI. (Note that for MPICH, AUTO corresponds to the RSA algorithm as this is the best option. In consequence, we only provide one row of charts for that MPI library.)
Figure 12 shows that pipelining also improves performance for legacy library versions. In this case, the results were obtained with OpenMPI 4.0. Compared with the version evaluated earlier in the paper (4.1), OpenMPI 4.0 does not allow to select a concrete algorithm for the MPI_Iallreduce CCP.

\section{Infiniband QDR}

Figure 13 demonstrates that the efficiency of pipelining is also visible in case the nodes of the target platform are connected via an older Infiniband QDR switch. In this case, the experiments were executed on a cluster with 15 nodes, each equipped with two Intel Xeon E5645 Westmere processors (6 cores each) and $48 \mathrm{~GB}$ of DDR3 RAM memory. The MPI libraries selected for this experiment were OpenMPI 4.0.1, MPICH 3.3.2, Intel MPI 2019. 

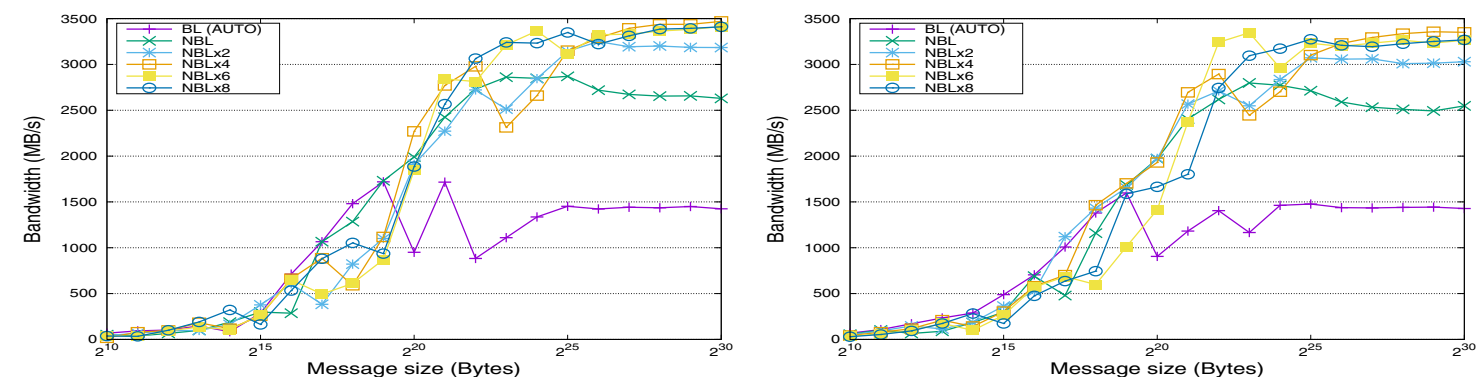

Fig. 12 Performance of the AUTO algorithm in OpenMPI (4.0) using 8 and 9 nodes/processes (left and right, respectively)
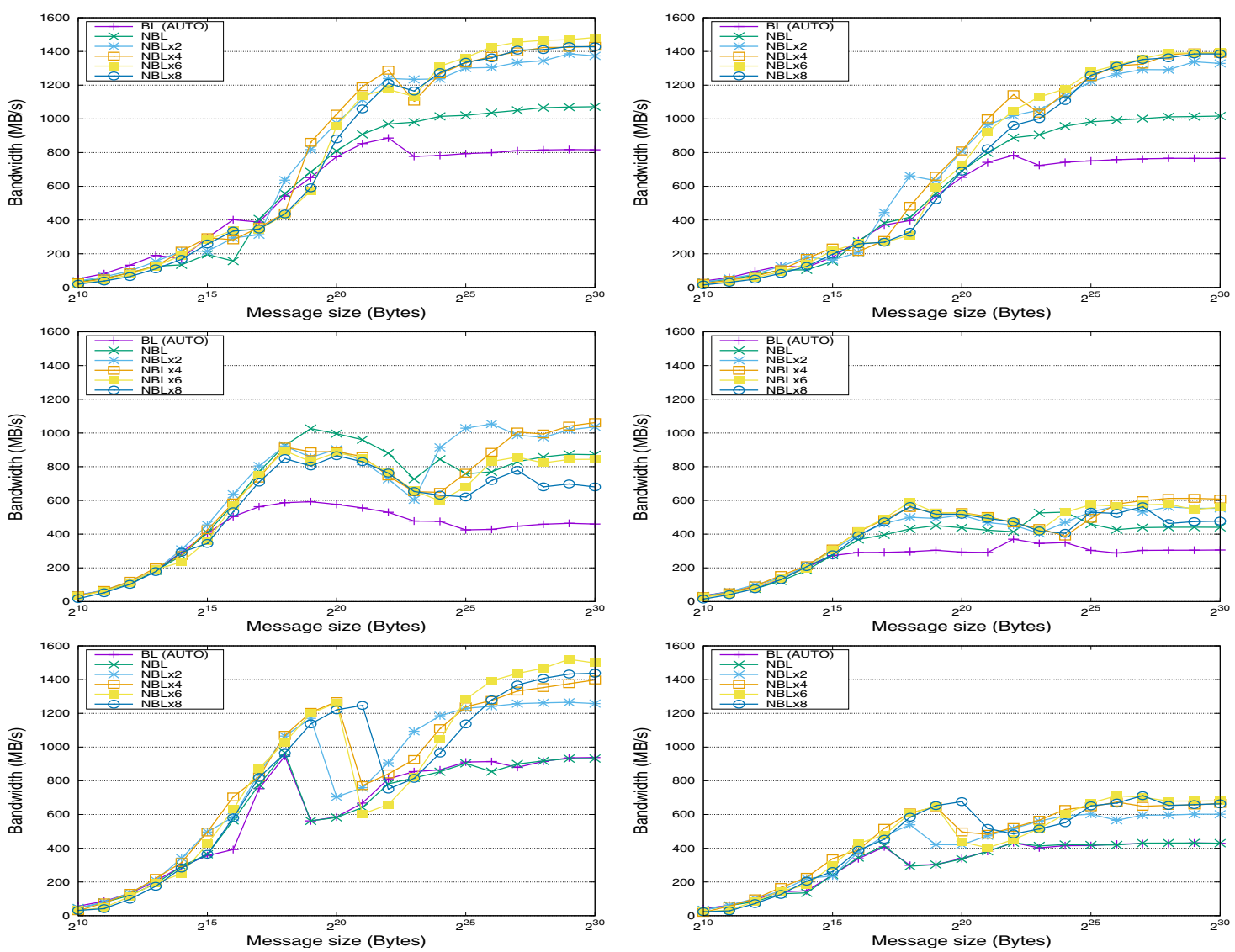

Fig. 13 Performance of the AUTO algorithm in OpenMPI 4.0 (top), and the AUTO and RNG algorithms in MPICH and Intel MPI (middle and bottom, respectively) using 8 and 15 nodes/processes (left and right, respectively)
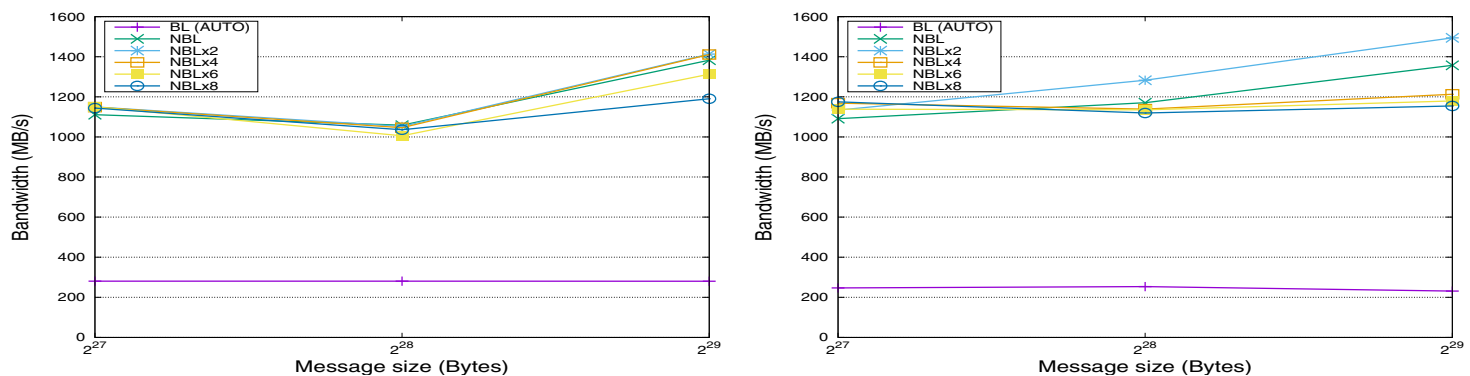

Fig. 14 Performance of the AUTO algorithm in OpenMPI (4.0) for large messages using 30 and 32 processes (left and right, respectively) 


\section{Network topology and processor family}

Finally, Fig. 14 reports the result of applying pipelining in the Marconi100 Supercomputer. ${ }^{7}$ This last experiment was executed with up to 32 nodes, where each cluster node was equipped with two IBM POWER9 AC922 processors (3.1 $\mathrm{GHz}, 16$ cores/processor) and $256 \mathrm{~GB}$ RAM each. (A node of Marconi also includes four NVIDIA Volta V100 GPUs, connected with NVLINK 2.0, and 16 GB.) The Infiniband network in this system is configured with a DragonFly topology (in all other experiments the topology was a FatTree). The results confirm that a significant benefit can, again, be gained from pipelining for large messages.

Acknowledgements This research was partially sponsored by project TIN2017-82972-R of the Spanish Ministerio de Ciencia, Innovación y Universidades and the Agencia Valenciana de la Innovación. Adrián Castelló was supported by the Juan de la Cierva-Formación project FJC2019-039222-I of the Ministerio de Ciencia, Innovación $y$ Universidades. Part of this work was executed on the Marconi100 supercomputing facility from CINECA Interuniversity Consortium HPC Department via the PRACE Preparatory Access project \#2010PA5531.

Author contributions All authors contributed to the study conception and design. Material preparation, data collection and analysis were performed by Adrián Castelló and Enrique S. Quintana-Ortí. The first draft of the manuscript was written by Adrián Castelló and was reviewed by Enrique S. Quintana-Ortí and Jose Duato. All authors commented on previous versions of the manuscript. All authors read and approved the final manuscript.

Funding Open Access funding provided thanks to the CRUE-CSIC agreement with Springer Nature. Project TIN2017-82972-R of the Spanish Ministerio de Ciencia, Innovación y Universidades. Agencia Valenciana de la Innovación. Juan de la Cierva-Formación project FJC2019-039222-I of the Ministerio de Ciencia, Innovación y Universidades PRACE Preparatory Access project \#2010PA5531.

Data availability Not applicable.

\section{Declarations}

Conflict of interest Not applicable.

Code availability The pipelined codes may be found at https://github. com/adcastel/collectives.

Open Access This article is licensed under a Creative Commons Attribution 4.0 International License, which permits use, sharing, adaptation, distribution and reproduction in any medium or format, as long as you give appropriate credit to the original author(s) and the source, provide a link to the Creative Commons licence, and indicate if changes were made. The images or other third party material in this article are included in the article's Creative Commons licence, unless indicated otherwise in a credit line to the material. If material is not included in the article's Creative Commons licence and your intended use is not permitted by statutory regulation or exceeds the permitted use, you will need to obtain permission directly from the copyright

\footnotetext{
7 https://www.hpc.cineca.it/hardware/marconi
}

holder. To view a copy of this licence, visit http://creativecommons. org/licenses/by/4.0/.

\section{References}

1. Abadi, M., Barham, P., Chen, J., Chen, Z., Davis, A., Dean, J., Devin, M., Ghemawat, S., Irving, G., Isard, M., et al.: TensorFlow: a system for large-scale machine learning. In: 12th USENIX Symposium on Operating Systems Design and Implementation (OSDI 16), pp. 265-283 (2016)

2. Alsmadi, I., Khreishah, A., Dianxiang, X.: Network slicing to improve multicasting in hpc clusters. Clust. Comput. 21(3), 1493-1506 (2018)

3. Awan, A.A., Bedorf, J., Chu, C.-H., Subramoni, H., Panda, D.K.: Scalable distributed DNN training using TensorFlow and CUDAaware MPI: characterization, designs, and performance evaluation (2018). arXiv: 1810.11112

4. Awan, A.A., Chu, C.-H., Subramoni, H., Panda, D.K.: Optimized broadcast for deep learning workloads on dense-GPU InfiniBand clusters: MPI or NCCL? In: Proceedings of the 25th European MPI Users' Group Meeting, pp. 1-9 (2018)

5. Ben-Nun, T., Hoefler, T.: Demystifying parallel and distributed deep learning: an in-depth concurrency analysis. ACM Comput. Surv. 52(4), 65:1-65:43 (2019)

6. Castelló, A., Catalán, M., Dolz, M.F., Mestre, J.I., Quintana-Ortí, E.S., Duato, J.: Evaluation of MPI Allreduce for distributed training of convolutional neural networks. In: 29th Euromicro International Conference on Parallel, Distributed and Networkbased Processing (PDP) (2021)

7. Castelló, A., Dolz, M.F., Quintana-Ortí, E.S., Duato, J.: Analysis of model parallelism for distributed neural networks. In: Proceedings of the 26th European MPI Users' Group Meeting, EuroMPI '19, New York, NY, USA (2019). Association for Computing Machinery

8. Castelló, A., Dolz, M.F., Quintana-Ortí, E.S., Duato, J.: Theoretical scalability analysis of distributed deep convolutional neural networks. In: 2019 19th IEEE/ACM International Symposium on Cluster, Cloud and Grid Computing (CCGRID), pp. 534-541 (2019)

9. Chan, E., Heimlich, M., Purkayastha, A., van de Geijn, R.: Collective communication: theory, practice, and experience. Concurr. Comput. 19(13), 1749-1783 (2007)

10. Clarke, L., Glendinning, I., Hempel, R.: The MPI message passing interface standard. In: Programming Environments for Massively Parallel Distributed Systems, pp. 213-218. Springer, Berlin (1994)

11. Deng, J., Dong, W., Socher, R., Li, L.-J., Li, K., Fei-Fei, L.: ImageNet: a large-scale hierarchical image database. In: 2009 IEEE Conference on Computer Vision and Pattern Recognition, pp. 248-255 (2009)

12. Hasanov, K., Lastovetsky, A.: Hierarchical redesign of classic MPI reduction algorithms. J. Supercomput. 73(2), 713-725 (2017)

13. He, K., Zhang, X., Ren, S., Sun, J.: Deep residual learning for image recognition. In: Proceedings of the IEEE Conference on Computer Vision and Pattern Recognition, pp. 770-778 (2016)

14. Google Inc. Tensorflow benchmarks. https://github.com/tensor flow/benchmarks

15. Ivanov, A., Dryden, N., Ben-Nun, T., Li, S., Hoefler, T.: Data movement is all you need: a case study on optimizing transformers (2020). arXiv:2007.00072

16. Kang, Q., Träff, J.L., Al-Bahrani, R., Agrawal, A., Choudhary, A., Liao, W.-k.: Full-duplex inter-group all-to-all broadcast 
algorithms with optimal bandwidth. In: Proceedings of the 25th European MPI Users' Group Meeting, pp. 1-10 (2018)

17. Kang, Q., Träff, J.L., Al-Bahrani, R., Agrawal, A., Choudhary, A., Liao, W-k: Scalable algorithms for MPI intergroup Allgather and Allgatherv. Parallel Comput. 85, 220-230 (2019)

18. Kim, Y., Choi, H., Lee, J., Kim, J.-S., Jei, H., Roh, H.: Towards an optimized distributed deep learning framework for a heterogeneous multi-gpu cluster. Clust. Comput. 23(3), 2287-2300 (2020)

19. Krizhevsky, A., Hinton, G., et al.: Learning multiple layers of features from tiny images. Technical report, Department of Computer Sciences, University of Toronto (2009)

20. Krizhevsky, A., Sutskever, I., Hinton, G.E.: ImageNet classification with deep convolutional neural networks. In: Proceedings of the 25th International Conference on Neural Information Processing Systems - Volume 1, NIPS'12, pp. 1097-1105, Curran Associates Inc. (2012)

21. Kurnosov, M., Tokmasheva, E.: Shared memory based mpi broadcast algorithms for numa systems. In: Russian Supercomputing Days, pp. 473-485. Springer, Berlin (2020)

22. Li, S., Hoefler, T., Chungjin, H., Snir, M.: Improved MPI collectives for MPI processes in shared address spaces. Clust. Comput. 17(4), 1139-1155 (2014)

23. Nguyen, T.T., Wahib, M., Takano, R.: Hierarchical distributedmemory multi-leader MPI_Allreduce for deep learning workloads. In: 2018 Sixth International Symposium on Computing and Networking Workshops (CANDARW), pp. 216-222. IEEE (2018)

24. Paszke, A., Gross, S., Massa, F., Lerer, A., Bradbury, J., Chanan, G., Killeen, T., Lin, Z., Gimelshein, N., Antiga, L., et al.: Pytorch: an imperative style, high-performance deep learning library. In: Advances in Neural Information Processing Systems, pp. 8026-8037 (2019)

25. Sergeev, A., Del Balso, M.: Horovod: fast and easy distributed deep learning in TensorFlow (2018). arXiv:1802.05799

26. Shalf, J.: HPC interconnects at the end of Moore's Law. In: 2019 Optical Fiber Communications Conference and Exhibition (OFC), pp. 1-3 (2019)

27. Simonyan, K., Zisserman, A.: Very deep convolutional networks for large-scale image recognition (2014). arXiv:1409.1556

28. Snir, M., Otto, S.W., Huss-Lederman, S., Walker, D.W., Dongarra, J.: MPI: The Complete Reference. The MIT Press, New York (1996)

29. Sze, V., Chen, Y.-H., Yang, T.-J., Emer, J.S.: Efficient processing of deep neural networks: a tutorial and survey. Proc. IEEE 105(12), 2295-2329 (2017)

30. Thakur, R., Rabenseifner, R., Gropp, W.: Optimization of collective communication operations in MPICH. Int. J. High Perform. Comput. Appl. 19(1), 49-66 (2005)

31. Worringen, J.: Pipelining and overlapping for MPI collective operations. In: 28th Annual IEEE International Conference on Local Computer Networks, 2003, pp. 548-557. IEEE (2003)

32. Zhao, Y., Wang, L., Wu, W., Bosilca, G., Vuduc, R., Ye, J., Tang, W., Xu, Z.: Efficient communications in training large scale neural networks. In: Proceedings of the on Thematic Workshops of ACM Multimedia 2017, pp. 110-116 (2017)

33. Zhong, D., Cao, Q., Bosilca, G., Dongarra, J.: Using advanced vector extensions avx-512 for mpi reductions. In: 27th European MPI Users' Group Meeting, pp. 1-10 (2020)
Publisher's Note Springer Nature remains neutral with regard to jurisdictional claims in published maps and institutional affiliations.

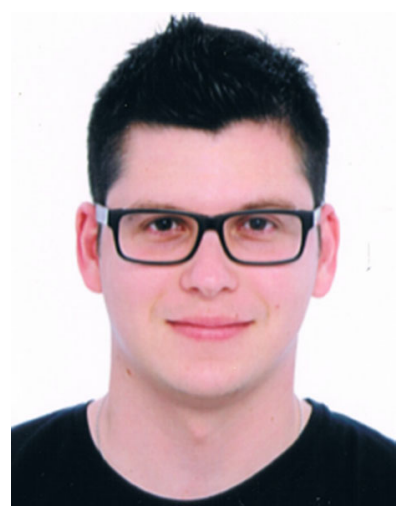

Adrián Castelló received his BS degree in computer science, the MS degree in advanced computer systems, and his Ph.D. degree in Computer Science from Universitat Jaume $I$ in 2011, 2013 and 2018, respectively. $\mathrm{He}$ is a Postdoc researcher at the Universitat Poitècnica de València and his research interests include highperformance computing, deep neural networks, programming models and distributed and shared memory systems.

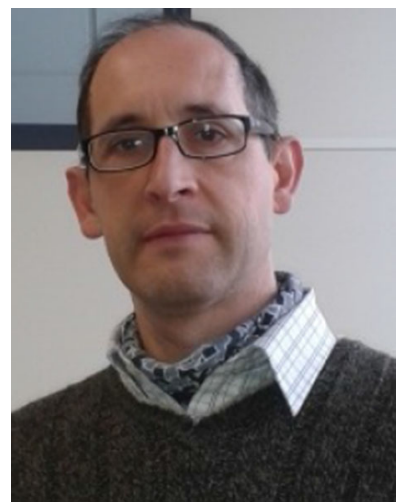

Enrique Quintana-Ortí received the bachelor and Ph.D. degrees in computer sciences from the Universitat Poitècnica de València, Spain, in 1992 and 1996, respectively. He is a Professor in Computer Architecture in the Universitat Poitècnica de València. Recently, he has participated in EU projects such as TEXT, INTERTWinE, EXA2GREEN and OPRECOMP. His current research interests include parallel programming, linear algebra, energy consumption, transprecision computing and bioinformatics as well as advanced architectures and hardware accelerators.

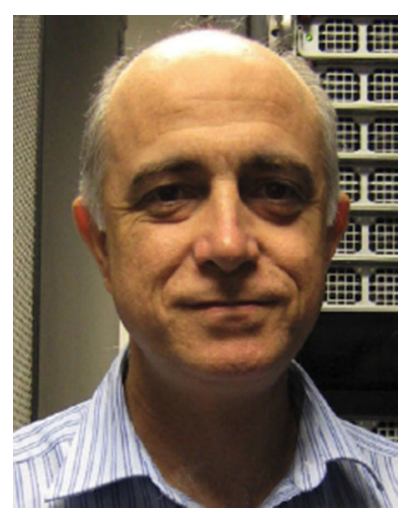

José Duato , Ph.D. in electrical engineering from the Technical University of Valencia (UPV), Spain. He is a professor in the Department of Computer Engineering at UPV. His current research interests include interconnection networks and multiprocessor architectures. $\mathrm{He}$ published more than 500 refereed papers that received more than 16,000 citations. He served as a member of the editorial board of IEEE TPDS, IEEE TC, and IEEE CAL, as general (co-) chair of ICPP 2001, HiPEAC 2019, and ISCA 2020, and as PC chair of HPCA 2004. He is a member of the Spanish Royal Academy of Sciences. 